\title{
PAPER
}

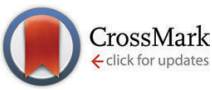

Cite this: New J. Chem., 2016, 40,2730

\section{A structural, spectroscopic and theoretical study of an o-vanillin Schiff base derivative involved in enol-imine and keto-amine tautomerism $\uparrow$}

\author{
Reinaldo Pis-Diez, ${ }^{a}$ Gustavo A. Echeverría, ${ }^{b}$ Oscar E. Piro, ${ }^{b}$ Jorge L. Jios ${ }^{c}$ and \\ Beatriz S. Parajón-Costa*a
}

\begin{abstract}
The potassium salt of the Schiff base obtained by condensation of 2-hydroxy-3-methoxybenzaldehyde (o-vanillin) and 2-aminoethanesulfonic acid (taurine) in a methanol potassium hydroxide solution was characterized by crystallographic, spectroscopic and DFT methods. The compound crystallizes in the monoclinic C2/c space group with $a=37.539(4), b=5.9129(4), c=26.321(5) \AA, \beta=121.10(2)^{\circ}$ and $Z=8$ molecules per unit cell. The crystallographic data reveal that two different anionic molecules, which constitute a tautomeric pair, coexist in the crystal. This finding is supported by FTIR, Raman and electronic measurements in methanol solution. The main crystallographic differences between the bonding structures occur along the shortest chemical path linking the oxygen and the nitrogen atom of the $\mathrm{OH}$ and $\mathrm{CN}$ group. Both tautomeric forms are stabilized by strong $\mathrm{O}-\mathrm{H} \cdots \mathrm{N}$ and $\mathrm{N}-\mathrm{H} \cdots \mathrm{O}$ intramolecular hydrogen bonds. Optimized geometrical parameters and calculated spectroscopic features obtained by DFT calculations show a very good agreement with the experimental data. Moreover, ${ }^{1} \mathrm{H}$ NMR, ${ }^{13} \mathrm{C}$ NMR and electronic measurements in DMSO solution show the prevalence of the enol-imine tautomer in this solvent.
\end{abstract}

Received (in Nottingham, UK) 6th May 2015,

Accepted 8th January 2016

DOI: 10.1039/c5nj01039j

www.rsc.org/njc

\section{Introduction}

Schiff bases are an interesting group of organic compounds that have been intensively investigated during the last few decades due to their relevance in chemistry, pharmacy, biochemistry and industry. They have an important role in the regulation of many biochemical processes and their properties as analytical reagents have been known for some time. ${ }^{1,2}$ During the last few years, cyclic and acyclic Schiff bases have been designed to be employed in the building of many molecular structures of interest in both applied

\footnotetext{
${ }^{a}$ CEQUINOR (CONICET, CCT-La Plata and UNLP), CC 962, La Plata, B1900AVV, Argentina. E-mail: beatrizp@quimica.unlp.edu.ar

${ }^{b}$ Departamento de Física, FCE (UNLP) and IFLP (CONICET, CCT-La Plata), CC 67, La Plata, B1900AVV, Argentina

${ }^{c}$ Unidad PLAPIMU-LASEISIC (UNLP-CIC), Departamento de Quimica, FCE (UNLP), 47 esq.115, La Plata, B1900AVV, Argentina

$\dagger$ Electronic supplementary information (ESI) available: Drawings of the molecular orbitals of the enol-imine and of the keto-amine form involved in the electronic transitions (Fig. S1 and S2, respectively). ${ }^{1} \mathrm{H}$ NMR, full spectrum (Fig. S3a) and expanded 2.7-7.2 ppm zone (Fig. S3b); ${ }^{13} \mathrm{C}$ NMR, full and expanded $45-175 \mathrm{ppm}$ zone (Fig. S4a and b, respectively). Tables of fractional coordinates and equivalent isotropic displacement parameters of the non-H atoms (Table S1), full bond distances and angles (Table S2), atomic anisotropic displacement parameters (Table S3), hydrogen atom positions (Table S4), and torsion angles (Table S5). CCDC 1056187. For ESI and crystallographic data in CIF or other electronic format see DOI: $10.1039 / \mathrm{c} 5 \mathrm{nj} 01039 \mathrm{j}$
}

and fundamental science. ${ }^{3}$ Specifically, in the field of coordination chemistry, aromatic Schiff bases containing nitrogen, oxygen and/or sulfur donor atoms and different functionalities are regarded as a valuable class of multidentantate chelating ligands because of the diversity of ways in which they can bind to different metal and oxometal cations. ${ }^{3-5}$ They are also considered to be useful materials for the design of some new and very promising pharmaceuticals due to their anticancer, antitubercular, antifungal, antibacterial and anticonvulsant activities, among other interesting properties. $^{2,4-6}$ They are also becoming increasingly important in material chemistry, catalysis and electrocatalysis ${ }^{7}$ and some of them have been used as effective corrosion inhibitors ${ }^{8}$ or as materials in electrochemical sensors for the detection of specific metal ions. ${ }^{9}$

Several $o$-hydroxy Schiff Bases exhibit interesting chromic properties and consequently they are considered to be suitable materials for the design of new optoelectronics devices, optical switches and biofunctional compounds. ${ }^{10,11}$ Their photo and thermochromic properties ${ }^{10-13}$ are related to their capacity to undergo prototropic tautomerism (Scheme 1), a process that can be studied in detail by combining different experimental and theoretical methods. ${ }^{14}$

Specifically, Schiff bases obtained by condensation of salicylaldheyde or substituted salicylaldheyde with derivatives of amines have been used as a model for the study of prototropic tautomerism..$^{13,15-20}$ As can be seen in Scheme 1, the equilibrium 


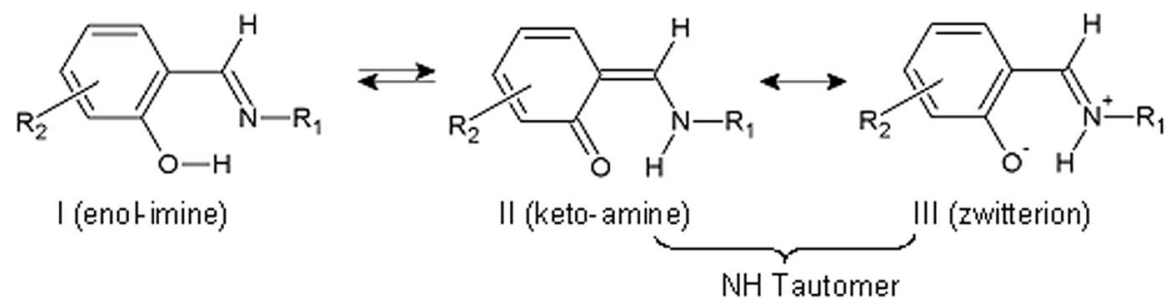

Scheme 1 Schematic representation of the tautomeric equilibrium in Schiff base compounds.

between the enol-imine (I) and the keto-amine (II) tautomer, in which the hydrogen is located at the nitrogen atom, is established by a reversible intramolecular proton transfer leading to a different $\pi$-electron distribution in the molecular structure of the compound. Moreover, tautomer (II) could be stabilized by resonance with its canonical zwitterionic form (III). ${ }^{15,16,19}$ Both structures are referred to as NH tautomers in Scheme 1.

On the other hand, the presence of the $-\mathrm{OH}$ group in the ortho position with respect to the $-\mathrm{C}(\mathrm{H})=\mathrm{N}$-group is a relevant factor that favors the existence of intramolecular hydrogen interaction between the hydroxyl of the aromatic ring and the nitrogen atom of the imine group. Depending on the tautomers, different types of intramolecular hydrogen interactions are possible (either $\mathrm{O}-\mathrm{H} \cdots \mathrm{N}$ for (I), $\mathrm{O} \cdots \mathrm{H}-\mathrm{N}$ for (II) or ${ }^{-} \mathrm{O} \cdots \mathrm{H}-\mathrm{N}^{+}$for (III)). ${ }^{16-19}$

Various studies performed on salicylaldehyde Schiff bases and their derivatives establish that most of them crystallize predominantly as enol-imine tautomers. However, the occurrence of the keto-amine form in the solid state, the coexistence of both enol/keto tautomers in the crystal, and a few examples of desmotropic systems are also reported. ${ }^{17-21}$ A broad study carried out on a series of methoxy-substituted salicylaldehyde Schiff bases establishes that the stabilization of each tautomeric form in the crystal is a function of the amine precursor and the position of the methoxy group on the salicylidene moiety. ${ }^{13}$ The compounds crystallize as cis-keto tautomers forming dimers through $\mathrm{O} \cdots \mathrm{H}-\mathrm{N}$ intermolecular hydrogen interactions, as enol tautomers or as an equimolar mixture of both tautomeric forms.

In connection with this type of compounds, we have reported the characterization by spectroscopic and theoretical methods of the acylhydrazone obtained by interaction of 3-methoxysalicylaldehyde (o-vanillin or, for short, $o$-HVa) with isoniazid (isonicotinic acid hydrazide), a mycobactericidal drug used for the treatment of tuberculosis. The compound, which was obtained as a chloride of the pyridinium salt, adopts the enol-imine form in the solid state without any evidence of tautomerism in solution. ${ }^{22}$ As a continuation of that research, in this work we report a study carried out using the Schiff base obtained from the condensation of $o$-vanillin with 2-aminoethanesulfonic acid (taurine, Tau from now on) in methanol solution containing potassium hydroxide. The compound, which was obtained as a potassium salt, will be named hereafter as $o$-VaTau $\left(\mathrm{R}_{1}=-\left(\mathrm{CH}_{2}\right)_{2} \mathrm{SO}_{3}{ }^{-}\right.$and $\mathrm{R}_{2}=$ $3-\mathrm{OCH}_{3}$ in Scheme 1). In order to attain structural and spectroscopic information of the molecular structure adopted by the $o$-VaTau compound, it has been characterized by elemental analysis, structural X-ray diffraction, FTIR, Raman, electronic and
RMN spectroscopy. Its conformational space was studied by DFT calculations and the lower-energy structures were compared with crystallographic data. The experimental vibrational modes and electronic transitions were assigned with the help of theoretical results.

Studies of transition metal complexes containing Schiff bases derived from $o$-vanillin and taurine or aminoacids, as ligands, have been described in the literature; ${ }^{23-26}$ however, to the best to our knowledge neither the crystal structure nor detailed studies of the spectroscopic properties of the $o$-VaTau compound have been reported previously.

\section{Results and discussion}

\section{Crystallographic structural results}

Crystal data and structural refinement results are summarized in Table 1. Fig. 1 shows an ORTEP $^{27}$ drawing of the crystal asymmetric unit content. The crystallographic data reveal that in the solid two different anionic molecules coexist as a tautomeric pair. The main differences between the bonding structures of both tautomers can be observed in the comparison of selected geometrical parameters shown in Table 2.

As can be seen in Scheme 1, the enol-imine form (I) contains a single $\mathrm{C}-\mathrm{O}$ bond and a double $\mathrm{C}=\mathrm{N}$ bond while the reverse is true for the keto-amine (II) tautomer. Consequently, it is reasonable to believe that by comparison of these experimental bond lengths is possible to distinguish between both tautomeric forms. ${ }^{13,20,28}$ Furthermore, as the NH tautomer is considered a resonance hybrid of the canonical structures (II) and (III) shown in Scheme 1, the prevalence of one form over the other could also be discerned from some of its characteristic geometrical parameters. ${ }^{15,29}$

In the $o$-VaTau crystal, one of the anionic molecules adopts the enol-imine structure (I) with a strong intramolecular O11-H $\cdots$ N1 hydrogen bond (Fig. 1 and Table 2). The C11-O11 and the $\mathrm{C} 17=\mathrm{N} 1$ bond distances are 1.346(4) $\AA$ and 1.273(4) $\mathrm{A}$, respectively, which are close to the values reported for the $\mathrm{C}-\mathrm{O}$ bond in phenols $(1.362 \AA)$ and for conjugated $\mathrm{C}=\mathrm{N}$ groups $(1.279 \AA) .{ }^{30}$ These bond lengths are also in agreement with those reported for several $o$-hydroxy Schiff bases that crystallize in the enol-imine tautomeric form. ${ }^{13,20,28}$ On the other hand, the second anionic molecule in the crystal adopts the keto-amine form (II). For this tautomer, the C21-O21 bond distance is shorter by $-0.075(6) \AA$ than the C11-O11 one and the C27-N2 bond distance is longer by +0.029 (6) $\AA$ than the corresponding $\mathrm{C} 17=\mathrm{N} 1$ bond length of tautomer (I) (see Table 2). 
Table 1 Crystal data and structure refinement results for o-VaTau

Empirical formula
Formula weight
Temperature
Wavelength
Crystal system
Space group
Unit cell dimensions

Volume

$Z$, density (calculated)

Absorption coefficient

$F(000)$

Crystal size

$\theta$-Range for data collection

Index ranges

Reflections collected Independent reflections Observed reflections

Completeness to $\theta=26.00^{\circ}$

Refinement method

Data/restraints/parameters

Goodness-of-fit on $F^{2}$

Final $R$ indices ${ }^{a}[I>2 \sigma(I)]$

$R$ indices (all data)

Largest diff. peak and hole

${ }^{a} R$ indices defined as: $R_{1}=\sum|| F_{\mathrm{o}}|-| F_{\mathrm{c}}|| / \sum\left|F_{\mathrm{o}}\right|, \mathrm{w} R_{2}=\left[\sum \mathrm{w}\left(F_{\mathrm{o}}{ }^{2}-F_{\mathrm{c}}{ }^{2}\right)^{2} /\right.$ $\left.\sum \mathrm{w}\left(F_{\mathrm{o}}^{2}\right)^{2}\right]^{1 / 2}$.

As can be seen in Table 2, additional differences between both tautomers are also observed in the Ci6-Ci1 and $\mathrm{Ci6}-\mathrm{Ci} 7$ bond lengths ( $i=1$ for the enol-imine and $i=2$ for the keto-amine form). Comparison of the geometric parameters shows that the formally double C16-C11 bond distance is shorter by $-0.032(7) \AA$ than the corresponding $\mathrm{C} 26-\mathrm{C} 21$ one and the single $\mathrm{C} 16-\mathrm{C} 17$ bond length is longer by +0.044(7) A than the C26-C27 distance. As a result, there are significant changes in the bonding structure of the $\mathrm{O} i 1-\mathrm{C} i 1-\mathrm{C} i 6-\mathrm{C} i 7-\mathrm{N} i$ skeleton of each tautomer. In fact, while the enol-imine form can be described by an O11-C11‥C16$\mathrm{C} 17=\mathrm{N} 1$ bonding scheme, the keto-amine structure can be better described as a delocalized $\mathrm{O} 21 \cdots \mathrm{C} 21 \cdots \mathrm{C} 26 \cdots \mathrm{C} 27 \cdots \mathrm{N} 2$ partial double bond structure where all the bond distances are perturbed by $\pi$ delocalization effects.
Furthermore, the phenol ring of tautomer (I) shows the usual delocalized resonant-bond structure with $\mathrm{C}-\mathrm{C}$ bond lengths in the 1.362(5)-1.409(5) ̊ range. The experimental results also show that the $\mathrm{Ci1}-\mathrm{Ci} 2$, Ci3-Ci4 and $\mathrm{Ci5}-\mathrm{Ci} 6$ bond distances in this tautomer are shorter than those determined for the keto-amine form, whereas the $\mathrm{Ci2}-\mathrm{Ci} 3$ and $\mathrm{Ci} 4-\mathrm{Ci} 5$ bond lengths show the opposite behavior (see Table 2). Thereby, the phenol ring of tautomer (II) can be better described in terms of localized single and double

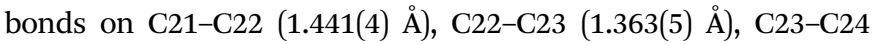

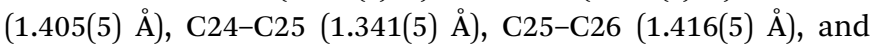
C26-C21 (1.427(5) Å). Additionally, the experimental N-H bond distance of $0.876 \AA$ is similar to those reported for several ortho hydroxy Schiff bases that crystallize in the keto-amine tautomeric form (II). ${ }^{28}$

Differences between both tautomers are also observed from the geometric features of intramolecular $\mathrm{O} 11-\mathrm{H} \cdots \mathrm{N} 1$ and O21 $\cdots \mathrm{H}-\mathrm{N} 2$ hydrogen bonds. ${ }^{13}$ The shorter $\mathrm{H} \cdots \mathrm{N} 1$ distance (1.699 $\AA$ ) with respect to the $\mathrm{H}$. . O21 distance (1.925 $\AA$ ) refers to a stronger intramolecular $\mathrm{H}$-bond for the enol-imine form. This finding is further supported by the shorter $\mathrm{O} 11 \cdots \mathrm{N} 1$ distance and the higher $\mathrm{O} 11-\mathrm{H} \cdots \mathrm{N} 1$ bond angle in tautomer (I) with respect to the corresponding $\mathrm{O} 21 \cdots \mathrm{N} 2$ distance and $\mathrm{O} 21 \cdots \mathrm{H}-\mathrm{N} 2$ bond angle in the keto-amine form, see Table 2.

Furthermore, crystallographic data show that three independent potassium ions are present in the solid. One of them (K1) is at a crystallographic general position in a compact eight-fold environment that includes the hydroxyl and the methoxyl oxygen atoms (K1 … distances of 2.983(3) and 2.806(3) A, respectively) and six neighboring sulfonate oxygen atoms (K1 . . O distances in the 2.680(3)-2.983(3) Å range). The other two potassium ions are at crystal special positions. One (K2) is on a two-fold axis in a distorted octahedral environment conformed by the phenolic oxygen $(d(\mathrm{~K} 2 \cdots \mathrm{O} 21)=2.805(2) \AA)$ and the sulfonate oxygen atoms

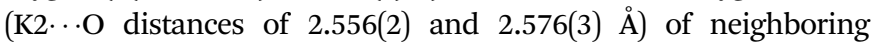
molecules. The third metal ion (K3) is sited on an inversion center in an eight-fold environment of neighboring sulfonate oxygen

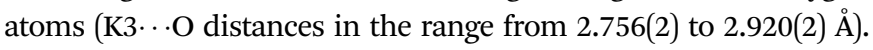

In Fig. 2, the powder X-ray diffraction pattern of $o$-VaTau is compared with the calculated one ${ }^{31}$ derived from the solid state molecular structure determined by single crystal X-ray diffraction.

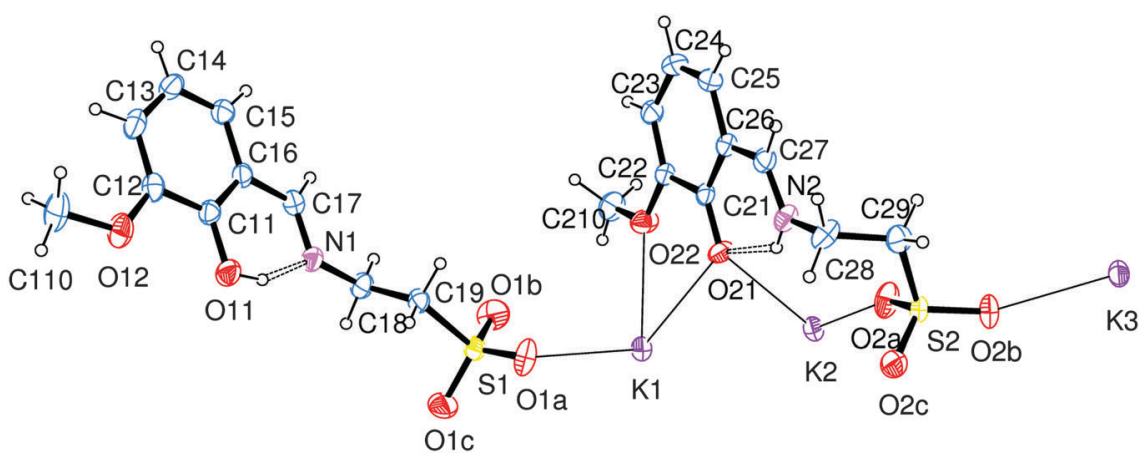

Fig. 1 View of o-VaTau showing the enol-imine (left) and the keto-amino (right) anionic molecules, the labeling of the non- $\mathrm{H}$ atoms and their displacement ellipsoids at the $30 \%$ probability level. Hydrogen-bonds are indicated by dashed lines and a few potassium-oxygen short contacts by thin lines. 
Table 2 Selected geometric parameters of the experimental enol-imine and keto-amine forms and of the calculated conformers I-a, II-b and II-C, see text. Bond distances $(d)$ are in angstroms and bond $(\alpha)$ and torsion $(\tau)$ angles are in degrees. See Fig. 1 for atom labels. The $i$ letter used to label atoms is equal to 1 for the enol-imine form and is equal to 2 for the keto-amine form

\begin{tabular}{llllll}
\hline & Enol-imine & $(\mathrm{I}-\mathrm{a})$ & Keto-amine & $(\mathrm{II}-\mathrm{b})$ & $(\mathrm{II}-\mathrm{c})$ \\
\hline$d(\mathrm{C} i 1-\mathrm{O} i 1)$ & $1.346(4)$ & 1.345 & $1.271(4)$ & 1.257 & 1.264 \\
$d(\mathrm{C} i 6-\mathrm{C} i 7)$ & $1.448(5)$ & 1.461 & $1.404(5)$ & 1.405 & 1.409 \\
$d(\mathrm{C} i 7-\mathrm{N} i)$ & $1.273(4)$ & 1.272 & $1.302(4)$ & 1.304 & 1.302 \\
$d(\mathrm{C} i 1-\mathrm{C} i 2)$ & $1.409(5)$ & 1.407 & $1.441(4)$ & 1.455 & 1.451 \\
$d(\mathrm{C} i 2-\mathrm{C} i 3)$ & $1.380(5)$ & 1.383 & $1.363(5)$ & 1.364 & 1.365 \\
$d(\mathrm{C} i 3-\mathrm{C} i 4)$ & $1.381(6)$ & 1.398 & $1.405(5)$ & 1.424 & 1.422 \\
$d(\mathrm{C} i 4-\mathrm{C} i 5)$ & $1.362(5)$ & 1.376 & $1.341(5)$ & 1.358 & 1.358 \\
$d(\mathrm{C} i 5-\mathrm{C} i 6)$ & $1.404(5)$ & 1.402 & $1.416(5)$ & 1.424 & 1.422 \\
$d(\mathrm{C} i 6-\mathrm{C} i 1)$ & $1.395(5)$ & 1.398 & $1.427(5)$ & 1.437 & 1.435 \\
$d(\mathrm{O} 11-\mathrm{H})$ & 0.907 & 0.997 & & & \\
$d(\mathrm{O} 11 \mathrm{H} \cdots \mathrm{N} 1)$ & 1.699 & 1.696 & & & \\
$d(\mathrm{~N} 2-\mathrm{H})$ & & & 0.876 & 1.026 & 1.029 \\
$d(\mathrm{O} 21 \cdots . \mathrm{HN} 2)$ & & & 1.925 & 1.901 & 1.770 \\
$d(\mathrm{O} i 1-\mathrm{N} i)$ & 2.549 & 2.598 & 2.634 & 2.667 & 2.613 \\
$\alpha(\mathrm{O} i 1-\mathrm{H}-\mathrm{N} i)$ & 154.1 & 148.3 & 136.7 & 128.9 & 136.3 \\
$\tau(\mathrm{C} i 6-\mathrm{C} i 7-\mathrm{N} i-\mathrm{C} i 8)$ & 178.9 & -179.2 & -178.2 & 178.8 & 179.6 \\
$\tau(\mathrm{C} i 7-\mathrm{N} i-\mathrm{C} i 8-\mathrm{C} i 9)$ & 115.5 & 107.7 & -99.7 & 126.2 & 114.2 \\
$\tau(\mathrm{N} i-\mathrm{C} i 8-\mathrm{C} i 9-\mathrm{S} i)$ & 166.8 & 171.3 & -73.2 & 74.0 & 177.2 \\
$\tau(\mathrm{C} i 1-\mathrm{C} i 2-\mathrm{O} i 2-\mathrm{C} i 10)$ & -171.3 & -177.4 & -172.6 & 179.2 & -177.3 \\
$\tau(\mathrm{C} i 5-\mathrm{C} i 6-\mathrm{C} i 7-\mathrm{N} i)$ & 179.6 & 178.4 & -176.4 & 179.7 & -178.9 \\
& & & & & \\
\hline
\end{tabular}

The figure shows that the material in the polycrystalline aggregation state employed in the solid state spectroscopic study reported here has the same crystal structure as its single crystal counterpart without significant contribution of impurities. Differences between experimental and theoretical integrated peak intensities could be due in part to polycrystalline preferential orientation.

\section{Optimized geometry}

Results of the conformational search in the solvent phase (methanol) indicate that the more stable conformations around the $\mathrm{C} 17-\mathrm{N} 1$ double bond correspond to a cis arrangement,

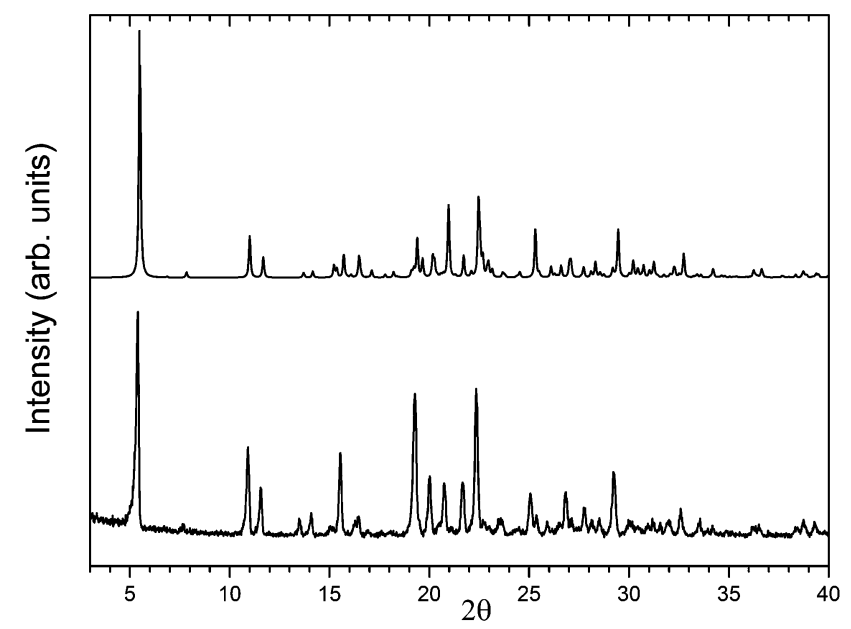

Fig. 2 Lower trace: experimental powder X-ray diffraction (PXRD) pattern of crystalline $O$-VaTau collected with CuK $\alpha$ radiation. Upper trace: PXRD pattern calculated from the solid state molecular structure of o-VaTau determined by single crystal X-ray diffraction methods. in which the $\mathrm{OH}$ group is close to the lone pair of the nitrogen atom, see Scheme 2 and Fig. 1 for labels.

Three cis conformations account for about $84 \%$ of the overall conformational space. On the other hand, the most stable trans conformation is found about $8 \mathrm{kcal} \mathrm{mol}^{-1}$ higher than the most stable cis conformer, thus making a negligible contribution to the molecular conformational space. One of the three more stable cis conformations corresponds to the experimental enol-imine form (I-a from now on, see Fig. 3) and contributes to about 59\% to the conformational space of the molecule. The other two cis conformers correspond to the experimental keto-amine form (II-b and II-c from now on, see Fig. 3) and accounts for the remaining $25 \%$ contribution. These different contributions are in excellent agreement with the coexistence of the two forms found in the solid phase by means of X-ray diffraction methods.

Table 2 compares some selected geometric parameters between the experimental forms and the calculated structures. It can be seen from the table that conformer I-a describes very well the enol-imine tautomer, confirming both that $\mathrm{C} 17-\mathrm{N} 1$ is a double bond and that $\pi$ electrons are fully delocalized in the $o$-HVa ring. The calculated $\mathrm{O} 11-\mathrm{H}$ bond distance is much larger than the experimental value by about $0.09 \AA$ but this fact is not reflected in a shorter $\mathrm{H} \cdots \mathrm{N} 1$ bond distance in conformer I-a with respect to the experimental value. Conformers II-b and II-c describe well the bond distances found in the keto-amine form but the calculated torsion angles for the taurine moiety differ widely from experimental values. This discrepancy could be attributed to strong lattice effects, which are not accounted for in the present calculations. Interestingly, the $\mathrm{C} 22-\mathrm{C} 23$ and C24-C25 bonds present an appreciable double character both in II-b and II-c suggesting that the $\mathrm{NH}$ tautomer adopts the keto-amine form (II) in the solvent phase. The calculated N2-H bond distance is much larger than the experimental value by about $0.05 \AA$ and this is accounted for by shorter $\mathrm{O} 21 \cdots \mathrm{H}$ bond distances in II-b and II-c with respect to the experimental distance.

Present results indicate that the enol-imine form does not undergo relevant structural changes when passing from the solvent phase to the solid phase. On the other hand, structural modifications in the keto-amine form are very important when the solid is formed from the methanol phase, especially in the taurine moiety and around the $\mathrm{O} 21 \cdots \mathrm{H}-\mathrm{N} 2$ subsystem.

\section{Vibrational spectroscopy}

The FTIR and Raman spectra of the $o$-VaTau in the $3300-400 \mathrm{~cm}^{-1}$ region are shown in Fig. 4. The solid-state vibrational properties of the compound are explained considering the coexistence of

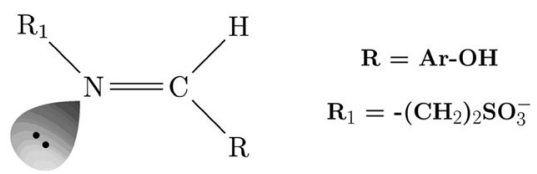

Scheme 2 The cis-trans isomerism around the N1-C17 double bond. For the cis arrangement, the $\mathrm{OH}$ group is located close to the lone pair of the nitrogen atom. 


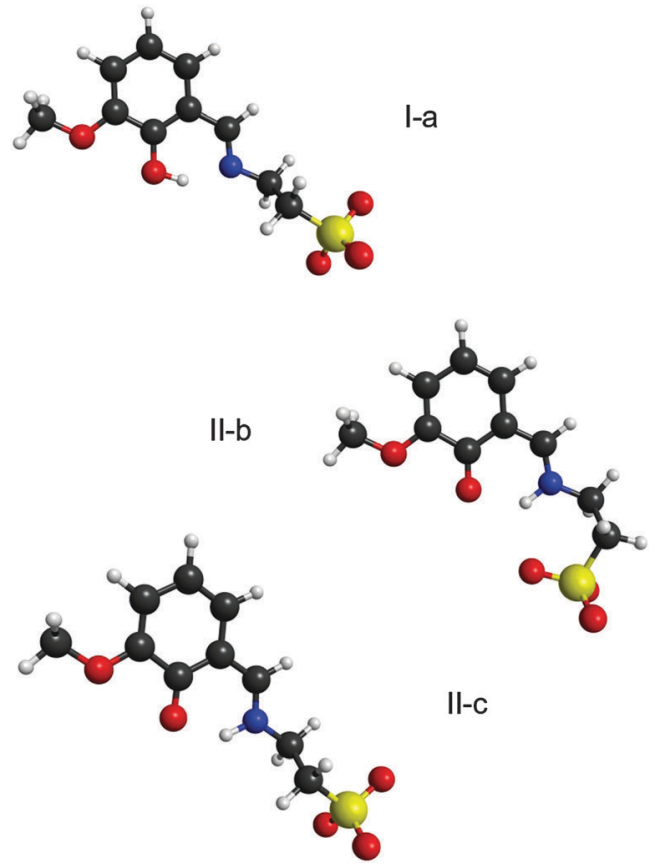

Fig. 3 Optimized geometries of the most stable conformations for the enol-imine form (I-a) and the keto-amine form (II-b and II-c) of O-OVaTau obtained at the M06-2X/cc-pVTZ level of theory, which includes solvent effects implicitly.

tautomers (I) and (II) in the crystal, in agreement with the crystallographic data. The observed frequencies were compared with those obtained for $o$-vanillin and taurine which adopt a zwitterionic configuration in the solid state, ${ }^{+} \mathrm{NH}_{3} \mathrm{CH}_{2} \mathrm{CH}_{2} \mathrm{SO}_{3}{ }^{-} \cdot{ }^{32}$

The assignments of the most important experimental and computed fundamental vibrational modes of $o$-VaTau are given in Table 3 and briefly discussed below (for atom numbering see Fig. 1). The assignment of some characteristic vibrational modes of taurine that is also included in Table 3 was done based on reported data ${ }^{33}$ and with the aid of calculated normal modes (unpublished results)

In the higher frequency region of the spectra, some characteristic stretching vibration modes are identified. The absorption bands due to the stretching of the $\mathrm{C}-\mathrm{H}$ of the aromatic ring and of the $\mathrm{C} i 7-\mathrm{H}$ appear in the $3100-2800 \mathrm{~cm}^{-1}$ region. The corresponding absorptions due to $\mathrm{CH}$ in-plane and out-of-plane deformations of the ring are observed in the $1400-1000 \mathrm{~cm}^{-1}$ and $800-700 \mathrm{~cm}^{-1}$ region, respectively. Calculation shows that they are coupled with other characteristic modes of the compound. The $\mathrm{C}-\mathrm{H}$ vibrational modes of the $-\mathrm{OCH}_{3}$ group of the $o$-vanillin precursor are conserved in the $o$-VATau spectra. ${ }^{34}$ For both proposed tautomers, the predicted values by theoretical calculations of these vibrations are in good agreement with the experimental results.

The formation of the proposed Schiff base is substantiated by the lack of the IR bands assigned to the $\mathrm{N}-\mathrm{H}$ stretching vibrations of the ${ }^{+} \mathrm{NH}_{3}$ group of taurine ${ }^{33}$ in the $3200-2800 \mathrm{~cm}^{-1}$ region and the $\mathrm{C}=\mathrm{O}$ stretching of the $o$-vanillin, ${ }^{34}$ as well as, by the presence of the strong and broad IR band centered at $1639 \mathrm{~cm}^{-1}$ (Ra: $1641 \mathrm{~cm}^{-1}$ and $1628 \mathrm{~cm}^{-1}$ ), which is the result

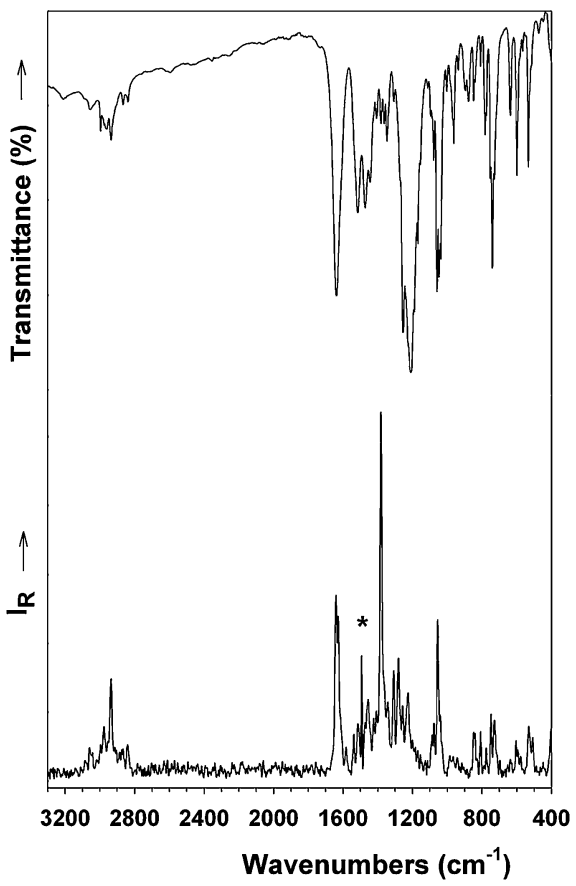

Fig. 4 Experimental IR and Raman spectra of o-VaTau in the $3300-400 \mathrm{~cm}^{-1}$ spectral range. (The Raman band at $1492 \mathrm{~cm}^{-1}\left(^{*}\right)$ originates from an instrumental noise).

of simultaneous vibrations of characteristic groups of both tautomers in the $1720-1560 \mathrm{~cm}^{-1}$ region. Although in this spectral region calculated values are slightly higher than experimental ones they allow us to distinguish between distinctive modes of each tautomeric form. The strong Raman signal at $1641 \mathrm{~cm}^{-1}$, predicted at $1706 \mathrm{~cm}^{-1}$, is attributed to the stretching of the $(\mathrm{C} 17=\mathrm{N} 1)$ bond of tautomer $(\mathrm{I}){ }^{35}$

Similarly, the Raman band at $1628 \mathrm{~cm}^{-1}$ is ascribed according to calculations to the asymmetric stretching of the $\mathrm{C} 26=\mathrm{C} 27-\mathrm{N} 2$ group that is coupled with the $\mathrm{C} 21=\mathrm{O} 21$ stretching mode, in tautomer (II). On the other hand, the formation of a six-membered ring (O21-C21‥C26-C27-N2H) in tautomer (II) by an intramolecular hydrogen bond $(\mathrm{O} 21 \cdots \mathrm{H}-\mathrm{N} 2)$ should affect the intensity and frequency of the N2-H stretching band as compared with the equivalent absorption of aromatic secondary amines, ${ }^{35}$ at about $3400 \mathrm{~cm}^{-1}$. Consequently, a weak IR band at $3221 \mathrm{~cm}^{-1}$ (calculated at $3274 \mathrm{~cm}^{-1}$ ) is assigned to this mode. Besides, calculations predict at $1534 \mathrm{~cm}^{-1}$ an absorption band due to the N2-H in-plane bending deformation coupled with the ring and the $(\mathrm{C} 21=\mathrm{O} 21)$ stretching modes. The weak Raman band at $1538 \mathrm{~cm}^{-1}$ is assigned tentatively to this deformation mode. The corresponding IR experimental band is probably overlapped with the broad absorption of media intensity at $1515 \mathrm{~cm}^{-1}$ ascribed to the in-plane-deformation of the $\mathrm{Ci} \mathrm{H}_{2}$ group of the taurine moiety in both tautomeric forms. Additionally, a weak IR band at $878 \mathrm{~cm}^{-1}$ is attributed by DFT calculations to the out-of-plane bending vibration of the $\mathrm{N} 2 \mathrm{H}$ group, which is coupled with the out-of-plane deformation of the ring.

According to the crystallographic data, the hydrogen of the $\mathrm{OH}$ group is involved in the formation of a six-membered ring 
Table 3 Assignment of some characteristics IR and Raman bands of the $\mathrm{O}-\mathrm{VaTau}$ compound. Experimental data of $\mathrm{O}-\mathrm{HVa}$ and taurine are included for comparison. Calculated frequencies for tautomers (I) and (II) are also presented. Frequencies are given in $\mathrm{cm}^{-1}$

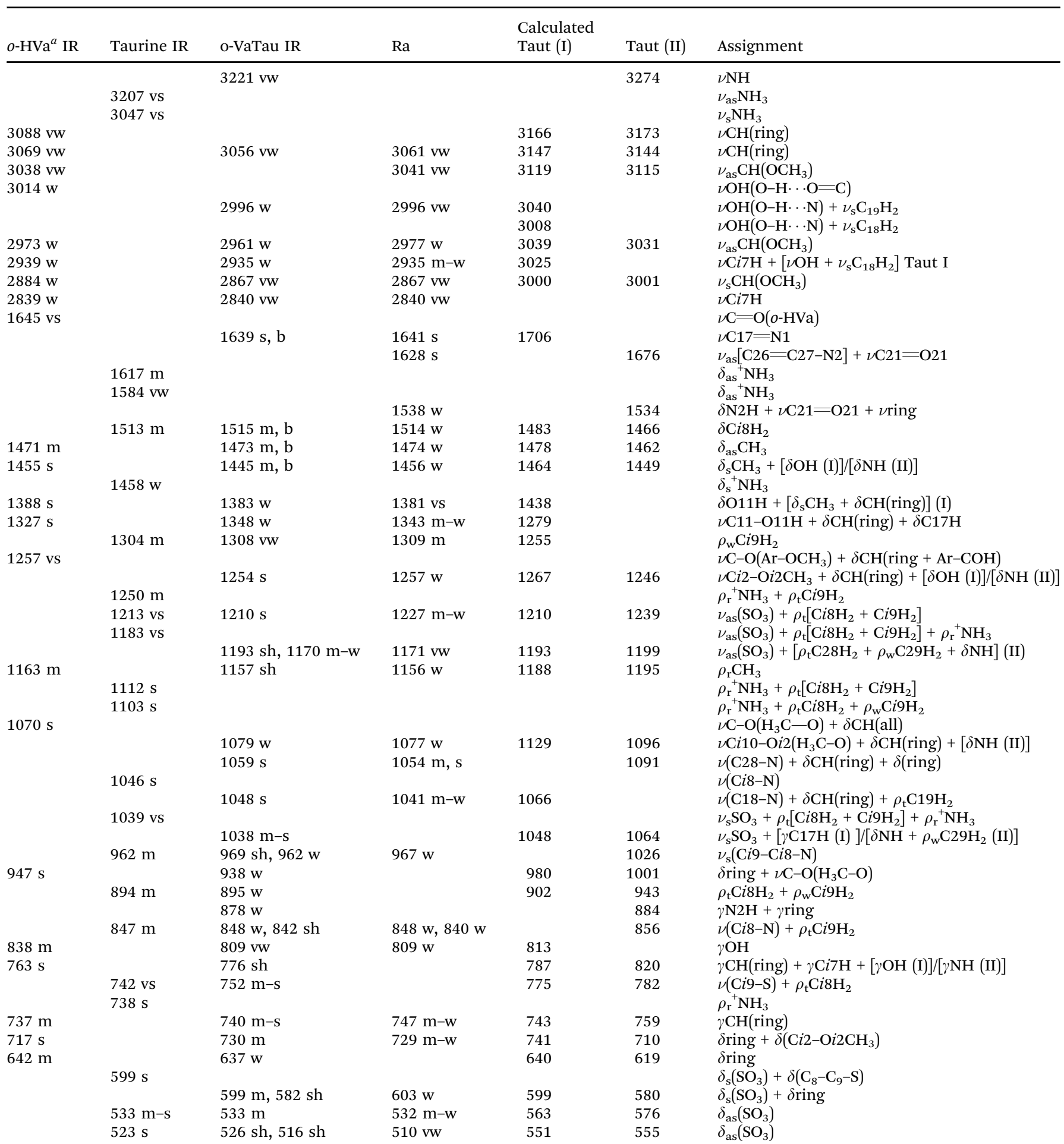

vs: very strong; s: strong; m: medium; w: weak; vw: very weak; sh: shoulder. Calculated frequencies are scaled by a factor of $0.982 . \nu$ : stretching; $\delta$ : in-plane deformation; $\gamma$ : out-of-plane deformation; $\rho_{\mathrm{t}}$ : twisting; $\rho_{\mathrm{w}}$ : wagging. $i=i, 1,2$ for taurine, tautomer I and tautomer II respectively. ${ }^{a}$ Data extracted from ref. 26. Notation [I]/[II] is used to indicate those individual modes of tautomer I and/or tautomer II that are coupled with vibrational modes, which are common to both forms.

(HO11-C11-C16-C17 = N1) by an intra-molecular hydrogen bond with N1. This type of interaction usually gives rise to a broad band of lower intensity than that expected for an intermolecular hydrogen bond and, sometimes, when this interaction becomes stronger the absorption due to the $\mathrm{O}-\mathrm{H}$ stretching mode is not detected. $^{35}$ In the present case, a weak IR band at $2996 \mathrm{~cm}^{-1}$, 
localized at a similar frequency to that reported for the $o$-HVA precursor, is ascribed to the stretching vibration of the hydroxyl group of tautomer $(\mathrm{I}), \nu(\mathrm{O} 11 \mathrm{H}) .^{34,35}$ The two calculated frequencies that described this vibration (at 3040 and $3008 \mathrm{~cm}^{-1}$ ) predict that the $\nu(\mathrm{O} 11 \mathrm{H})$ mode is coupled with the symmetric stretching of the $\mathrm{C}_{19 \mathrm{H}_{2}}$ and $\mathrm{C}_{18 \mathrm{H}_{2}}$ groups of the taurine moiety, respectively. On the other hand, the relatively high frequency of the $-\mathrm{O} 11 \mathrm{H}$ out-of plane bending vibration (IR: $809 \mathrm{~cm}^{-1}$, calculated $813 \mathrm{~cm}^{-1}$ ) agrees well with the predicted correlation for this type of compound between the strength of the hydrogen interaction and the expected frequency values for the $\nu(\mathrm{OH})$ and $\gamma(\mathrm{OH})$ modes. $^{36}$ The calculated harmonic vibration frequencies indicate that the $\delta(\mathrm{O} 11 \mathrm{H})$ and the $\nu(\mathrm{C} 11-\mathrm{O} 11 \mathrm{H})$ modes at 1383 and $1348 \mathrm{~cm}^{-1}$, respectively, are mixed with other characteristic vibrations, as can be observed in Table 3 .

In connection with the taurine moiety, in the $o$-VaTau compound all the bands corresponding to the ${ }^{+} \mathrm{NH}_{3}$ vibrational modes are absent. In addition, the characteristic absorption bands for the $-\mathrm{Ci} 8 \mathrm{H}_{2},-\mathrm{Ci} \mathrm{H}_{2}$, and the $-\mathrm{SO}_{3}{ }^{-}$vibrations are observed at the expected regions. ${ }^{33,35}$ The broad and strong IR absorption band in the $1300-1100 \mathrm{~cm}^{-1}$ region appears to be composed of several overlapping bands, which are attributed to different vibrational modes of the Schiff base compound (Table 3). In this region, the strong IR band at $1210 \mathrm{~cm}^{-1}$ with a shoulder at $c a .1228 \mathrm{~cm}^{-1}$ and the band of media intensity at $1170 \mathrm{~cm}^{-1}$ with a shoulder at $1193 \mathrm{~cm}^{-1}$ are attributed to the asymmetric stretching vibrations of the $\mathrm{SO}_{3}{ }^{-}$group. The symmetric stretching of this group gives rise to the band of media intensity located at $1038 \mathrm{~cm}^{-1}$. The IR absorptions in the $600-500 \mathrm{~cm}^{-1}$ region are assigned to the symmetric and asymmetric deformation of the $\mathrm{SO}_{3}{ }^{-}$in accordance with the corresponding ones in the IR spectrum of taurine. Calculations show that these modes are not pure, but they contain contributions from other distinctive modes of each tautomer.

\section{Electronic spectroscopy}

The electronic spectrum of a $5 \times 10^{-5} \mathrm{M}$ methanol solution of $o$-VaTau was registered in the $200-800 \mathrm{~nm}$ spectral range. The wavelengths corresponding to the observed maxima are listed in Table 4. The calculated transition energies, converted to wavelengths, for both the enol form (I-a conformer) and the keto form (II-b conformer) are also listed in Table 4. As the calculated transition energies and oscillator strengths for the II-c conformer are very similar to that obtained for II-b, they are neither included in Table 4 nor considered in the discussion.
In Fig. 5 the experimental spectrum is compared with the simulated one from the calculated transition energies and oscillator strengths.

As can be seen in the figure the experimental spectrum is characterized by a strong band at $222 \mathrm{~nm}$ and other four bands of lower intensities located at $262 \mathrm{~nm}, 292 \mathrm{~nm}, 334 \mathrm{~nm}$ and $413 \mathrm{~nm}$. Moreover, a shoulder is observed at $250 \mathrm{~nm}$. The molecular orbitals of the enol-imine and of the keto-amine forms involved in the electronic transitions are shown in Fig. S1 and S2 in the ESI. $\dagger$

It can be seen from Table 4 and Fig. 5 that the experimental spectrum of $o$-VaTau is well described by the theoretical calculations. The band located at $222 \mathrm{~nm}$ is assigned to a transition from the HOMO to the LUMO+1, which is present in both tautomers. Both, the HOMO and the LUMO+1, are localized at the $o$-HVa moiety of $o$-VaTau, see Fig. S1 and S2 in the ESI. $\dagger$ The band at $262 \mathrm{~nm}$ and the shoulder at $250 \mathrm{~nm}$ are assigned to transitions that originated in the enol-imine form of $o$-VaTau, although with opposite relative intensities. The band at $292 \mathrm{~nm}$, on the other hand, is assigned to a transition that involves molecular orbitals belonging to the keto-amine form. It can be observed in Fig. S1 and S2 (ESI $\dagger$ ) that both the HOMO-1 and the LUMO are localized at the $o$-HVa moiety with some contribution from the electronic lone pair of the nitrogen atom. The HOMO-3 is mainly localized at the nitrogen atom. Interestingly, II-b also exhibits a transition at $249 \mathrm{~nm}$ from the HOMO-3 to the LUMO, but the oscillator strength in this case is negligible. Finally, the bands at $334 \mathrm{~nm}$ (I-a) and $413 \mathrm{~nm}$ (II-b) are assigned to a transition from the HOMO to the LUMO in the enol-imine and in the keto-amine tautomeric forms, respectively.

Experimental and theoretical results point out that in methanol solution the compound exists as a mixture of the enol-imine and the keto-amine tautomers in equilibrium. These results are in agreement with previous studies on similar systems which show that the stabilization of the keto-amine tautomer is achieved in protic polar solvents. The presence of this tautomer in solution is mainly ascribed to a band at higher frequency than those attributed to the enol-imine tautomer $(\lambda \geq 400 \mathrm{~nm}) \cdot{ }^{18,20,37-39}$ In contrast, the electronic spectrum of $o$-VaTau in dimethylsulfoxide (DMSO) shows only two dominant absorption bands in the $250-800 \mathrm{~nm}$ spectral range. The first one lies close to the solvent cut-off, at $262 \mathrm{~nm}$, and the second at $330 \mathrm{~nm}$. The absence of the characteristic absorption of the keto-amine form indicates that, in this aprotic solvent, the compound is present predominantly in the enol-imine form,

Table 4 Experimental and calculated wavelengths for o-VaTau, in $\mathrm{nm}$. Calculated wavelengths (Calc) and oscillator strengths (Osc str), in arbitrary units, are provided for $\mathrm{I}-\mathrm{a}$ and II-b. The proposed assignment, with percentage contributions in parentheses, is also given

\begin{tabular}{|c|c|c|c|c|c|c|}
\hline Exp & \multicolumn{3}{|l|}{$\mathrm{I}-\mathrm{a}$} & \multicolumn{3}{|l|}{ II-b } \\
\hline 222 & 216 & 0.6545 & HOMO $\rightarrow$ LUMO+1 (91\%) & 229 & 0.2994 & HOMO $\rightarrow$ LUMO+1 (90\%) \\
\hline 262 & 260 & 0.0286 & HOMO $-3 \rightarrow$ LUMO $(88 \%)$ & & & \\
\hline 292 & & & & 272 & 0.3561 & HOMO-1 $\rightarrow$ LUMO $(91 \%)$ \\
\hline 334 & 312 & 0.0735 & HOMO $\rightarrow$ LUMO (97\%) & & & \\
\hline
\end{tabular}




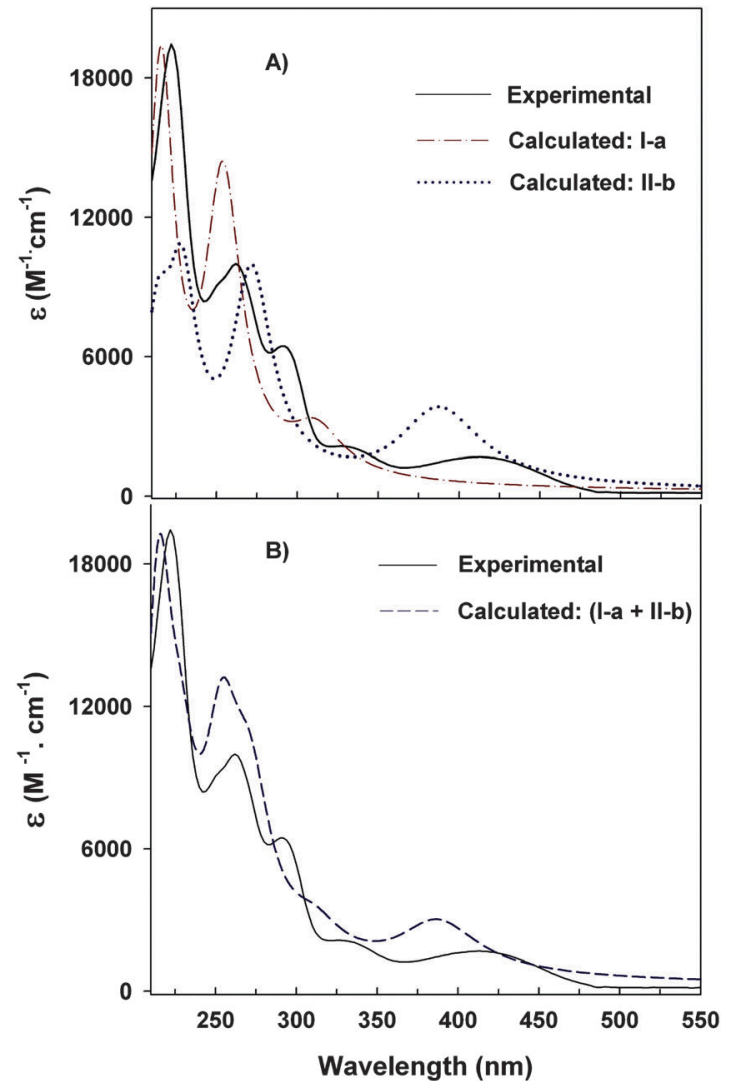

Fig. 5 Comparison of the electronic spectrum of a methanol solution $\left(5 \times 10^{-5} \mathrm{M}\right)$ of $O$-VaTau with: (A) the calculated spectra for I- $a$ and for II-b, (B) the statistically-averaged simulated spectrum, obtained as a combination of the calculated spectra of I-a (59\%) and II-b (25\%). Simulated spectra are normalized with respect to the experimental one to ease the visualization.

as confirmed by NMR measurements realized in $\mathrm{d}_{6}$-DMSO, and in agreement with previous studies on similar systems which establish the predominance of this tautomer in aprotic solvents. ${ }^{17,20}$

\section{NMR spectroscopy}

The ${ }^{1} \mathrm{H}$ and ${ }^{13} \mathrm{C}$ NMR signals for $o$-VaTau $\left(\mathrm{d}_{6}\right.$-DMSO) were unambiguously assigned on the basis of the multiplicities, coupling constant values and intensities $\left({ }^{1} \mathrm{H}\right)$ and bidimensional spectra. The ${ }^{13} \mathrm{C}$ NMR assignments of the protonated carbons followed from the analysis of the one-bond heteronuclear correlation data using the Heteronuclear Single Quantum Coherence pulse diagrams (HSQC). The quaternary carbons were unequivocally determined from the long-range correlation responses observed with the previously assigned ${ }^{1} \mathrm{H}$ signals in the Heteronuclear Multiple Bond Correlation (HMBC) diagrams. For all other carbon atoms, well-established increment rules were used. ${ }^{40}$

The ${ }^{1} \mathrm{H}$ NMR (Fig. S3a and S3b, ESI $\dagger$ ) and ${ }^{13} \mathrm{C}$ NMR spectra (Fig. S4a and $\mathrm{S} 4 \mathrm{~b}$, ESI $\dagger$ ) of $o$-VaTau in the aprotic solvent $\mathrm{d}_{6}$-DMSO show that the enol-imine/keto-amine equilibrium is shifted to the enol-imine form in concordance with electronic measurements in the same solvent. No signals belonging to the keto-amine tautomer are detected in this solvent. Table 5 displays
Table $5{ }^{1} \mathrm{H}(200.1 \mathrm{MHz})$ and ${ }^{13} \mathrm{C}(50.3 \mathrm{MHz})$ NMR chemical shifts, in ppm, for 0 -VaTau $\left(d_{6}\right.$-DMSO). See Fig. 1 for atom labeling

\begin{tabular}{lll}
\hline $\mathrm{C} / \mathrm{H}$ & $\delta_{\mathrm{C}}$ & $\delta_{\mathrm{H}}$ \\
\hline $\mathrm{O}-\mathrm{H}$ & & $13.71(\mathrm{~s})$ \\
$\mathrm{CH}_{3}$ & 55.7 & $3.77(\mathrm{~s})$ \\
11 & 152.8 & \\
12 & 148.3 & $6.93-7.04(\mathrm{~m})$ \\
13 & 114.6 & $6.75(\mathrm{t}, J=8 \mathrm{~Hz})$ \\
14 & 117.1 & $6.93-7.04(\mathrm{~m})$ \\
15 & 123.3 & \\
16 & 118.1 & $8.53(\mathrm{~s})$ \\
17 & 166.2 & $3.85(\mathrm{t}, J=7 \mathrm{~Hz})$ \\
18 & 54.3 & $2.79(\mathrm{t}, J=7 \mathrm{~Hz})$ \\
19 & 51.8 &
\end{tabular}

The signal multiplicity and coupling constants $(J$ in Hertz) are in parentheses.

the ${ }^{1} \mathrm{H}$ and ${ }^{13} \mathrm{C}$ NMR chemical shifts for the compound, along with the signal assignment.

The phenolic proton is observed at $13.71 \mathrm{ppm}$ and evidences its strong intramolecular hydrogen bond interaction, $\mathrm{O}-\mathrm{H} \cdots \mathrm{N}$, with the lone pair of the $\mathrm{sp}^{2}$ hybridized nitrogen atom of the imino group. ${ }^{41}$ Furthermore, the $\mathrm{H}-17$ (the hydrogen bonded to $\mathrm{C} 17$ in the $\mathrm{HC}=\mathrm{N}$ moiety) is observed as a singlet at $8.53 \mathrm{ppm}$. The signals at 7.00-6.75 ppm are indicative of aromatic protons ( $\mathrm{H}-14$ is a triplet composed of two ortho couplings with $\mathrm{H}-13$ and $\mathrm{H}-15)$ and a value of $\mathbf{1 5 2 . 8} \mathrm{ppm}$ is typical for aromatic carbon atoms directly attached to $\mathrm{OH}$ groups. The corresponding protons in the keto-amine tautomer should have cis coupling multiplicities (cicloalcadiene portion) whereas $\mathrm{H}-17$ should show a doublet as the result of the vicinal coupling with the amino proton. Besides, the ${ }^{13} \mathrm{C}$ NMR chemical shifts at around $180 \mathrm{ppm}$ for carbonyl carbon atoms ${ }^{17,41}$ and the ${ }^{1} \mathrm{H}$ NMR characteristic signal of the amino proton with intramolecular interaction $\mathrm{N}-\mathrm{H} \cdots \mathrm{O}=\mathrm{C}$, between 10 and $12 \mathrm{ppm}(\mathrm{eg}$. $11.55 \mathrm{ppm}$ in an acyclic $\beta$-aminoenone), ${ }^{42}$ are missing in the respective spectra.

\section{Conclusions}

A structural and spectroscopic study of the potassium salt of the Schiff base obtained by condensation of 2-hydroxy-3-methoxybenzaldehyde and 2-aminoethanesulfonic acid is performed. To support the interpretation of the experimental results, the analysis is complemented with a DFT theoretical study. Structural X-ray diffraction methods reveal the coexistence of two different anionic molecules in the solid phase that are stabilized by strong $\mathrm{O}-\mathrm{H} \cdots \mathrm{N}$ and $\mathrm{N}-\mathrm{H} \cdots \mathrm{O}$ intramolecular hydrogen bonds. Accordingly, when the conformational space is explored in the solvent phase three lowest energy conformers are found, two of them correspond to the keto-amine form and the remaining one corresponds to the enol-imine form. These findings are in agreement with experimental data that show that the anionic molecules in the crystal can be described best as a mixture of both enol/keto tautomeric forms. New absorptions and splitting or broadening of some characteristic vibrational bands provide additional evidence of the coexistence of both tautomers in the solid state. 
UV-vis measurements reveal that in solution the preferred tautomeric form depends on the solvent. Studies performed in DMSO by electronic and RMN spectroscopies show that the enol-imine tautomer predominates. In contrast, a mixture in equilibrium of both the enol and the keto forms is present in a protic polar solvent, methanol. Calculated low-energy transitions describe very well the experimental spectra and allow the full assignment of the observed absorption bands.

\section{Experimental}

\section{Materials and the general procedure for synthesis}

All reagents were of analytical grade. 2-Hydroxy-3-methoxybenzaldehyde (o-vanillin, Sigma), 2-aminoethanesulfonic acid (taurine, Sigma), methanol and dimethylsulfoxide (Merck) were used as purchased without further purification. The potassium salt of the Schiff base was prepared according to a known general procedure for this type of compound. ${ }^{26} \mathrm{~A}$ methanol solution $(80 \mathrm{~mL})$ containing $10 \mathrm{mmol}(1.25 \mathrm{~g})$ of taurine and $10 \mathrm{mmol}(0.56 \mathrm{~g})$ of potassium hydroxide was dropwise added to a solution of $10 \mathrm{mmol}(1.52 \mathrm{~g})$ of $o$-vanillin in methanol $(10 \mathrm{~mL})$ with continuous stirring. The initial colorless solution turned immediately to bright gold yellow. The mixture was then stirred for 45 minutes at room temperature to give a yellow precipitate of the $o$-VaTau compound. The system was left in digestion for one hour and then the solid was filtered off, washed with small portions of methanol and then dried in air. After one week, by further precipitation from the filtrate at room temperature, pale yellow single crystals, suitable for X-ray structural analysis, were obtained. Anal. Calcd. for $\mathrm{C}_{20} \mathrm{H}_{24} \mathrm{~K}_{2} \mathrm{~N}_{2} \mathrm{O}_{10} \mathrm{~S}_{2}$ : C, 40.39; H, 4.06; N, 4.70. Found: C, 39.98; H, 4.37; N, 4.92\%.

\section{Physicochemical methods}

Elemental analysis (C, H, N) was performed using a Carlo Erba EA 1110 analyzer. FTIR spectra $\left(4000-400 \mathrm{~cm}^{-1}\right)$ were recorded using the KBr pellet technique on an FTIR Bruker Equinox 55 instrument. Raman spectra were obtained on a Bruker IFS 66 FTIR spectrophotometer, using the FRA 106 Raman accessory and the $1064 \mathrm{~nm}$ line of a Nd:YAG solid state laser for excitation. The electronic absorption spectrum was recorded on freshly prepared methanol solutions in the $200-800 \mathrm{~nm}$ spectral range. It was recorded on a Hewlett-Packard 8452-A diode array spectrometer, using $10 \mathrm{~mm}$ quartz cells. The ${ }^{1} \mathrm{H}$ NMR $(200.1 \mathrm{MHz})$ and ${ }^{13} \mathrm{C}(50.3 \mathrm{MHz})$ spectra were recorded at $298 \mathrm{~K}$ on a Bruker AC 200 spectrometer. The sample was dissolved in $\mathrm{d}_{6}$-DMSO in a $5 \mathrm{~mm}$ NMR tube. Chemical shifts, $\delta$, for ${ }^{1} \mathrm{H}$ and ${ }^{13} \mathrm{C}$ are given in parts per million relative to tetramethylsilane (TMS; $\delta=0 \mathrm{ppm}$ ). Calibration was made with the solvent residual peaks as references $(2.50$ and $39.52 \mathrm{ppm}$ for hydrogen and carbon, respectively). Coupling constants, $J$, are reported in Herz.

\section{X-ray crystallography}

\section{Powder X-ray diffraction (PXRD)}

The PXRD pattern of solid state $o$-VaTau was obtained on a PANalytical X'Pert PRO diffractometer, using $\mathrm{CuK} \alpha$ radiation $(\lambda=1.5406 \AA$ A) from an X-ray tube operated at $40 \mathrm{kV}$ and $40 \mathrm{~mA}$. The X-ray diffraction pattern was collected in the $2^{\circ} \leq 2 \theta \leq 60^{\circ}$ range, with $0.02^{\circ}$ step width and 1 s counting time per step employing the Bragg-Brentano $\theta-\theta$ geometry, a scintillation counter, and an exit beam graphite monochromator.

\section{Single crystal diffraction}

The measurements were performed on an Oxford Xcalibur Gemini, Eos CCD diffractometer with graphite-monochromated $\operatorname{MoK} \alpha(\lambda=0.71073 \AA)$ radiation. X-ray diffraction intensities were collected ( $\omega$ scans with $\theta$ and $\kappa$-offsets), integrated and scaled with CrysAlisPro $^{43}$ suite of programs. The unit cell parameters were obtained by least-squares refinement (based on the angular settings for all collected reflections with intensities larger than seven times the standard deviation of measurement errors) using CrysAlisPro. Data were corrected empirically for absorption employing the multi-scan method implemented in CrysAlisPro. The structure was solved by direct methods with SHELXS of the SHELX suit of programs ${ }^{44}$ and the molecular model developed by alternated cycles of Fourier methods and full-matrix least-squares refinement with SHELXL of the same package. All the hydrogen atoms were located in a Fourier difference map phased on the heavier atoms and refined at their found positions with isotropic displacement parameters. Both methyl groups converged to staggered conformations.

\section{Computational methods}

The conformational space of $o$-VaTau was explored using the PM $^{45}$ method as implemented in MOPAC. ${ }^{46}$ The cis-trans isomerism around the $\mathrm{N} 1-\mathrm{C} 17$ double bond was first considered (see Scheme 2 and Fig. 1 for labels). For every geometric isomer several starting structures were obtained by selected modifications of C17-N1-C18-C19, N1-C18-C19-S and C11-C12-O12-C110 dihedral angles.

The structures obtained experimentally from X-ray diffraction methods were also used as the starting point. All the structures were optimized at the PM6 level of theory and the resulting geometries were analyzed to discard duplicated structures. The geometries were further optimized using the hybrid meta-GGA exchange-correlation functional M06-2X, ${ }^{47,48}$ with the cc-pVTZ basis set for all atoms. After optimization, the Hessian matrix of the energy with respect to the nuclear coordinates was diagonalized and its eigenvalues were used to verify whether the geometries were local minima or saddle points on the potential energy surface of the molecules. When the eigenvalues of the Hessian matrix were all positive, they were converted to harmonic vibrational frequencies and some thermodynamic properties were calculated. The contribution of every conformer to the overall conformation space was calculated using the Maxwell-Boltzmann classical statistics with the Gibbs free energy at $298 \mathrm{~K}$ as the energy parameter. Vibrational frequencies were scaled by a factor of 0.982 to ease the comparison with experimental values. ${ }^{47}$

The electronic transitions of $o$-VaTau were calculated within the framework of the time-dependent density functional theory. ${ }^{49}$ In this case, the hybrid GGA exchange-correlation functional 
PBE0 $^{50}$ was used with the cc-pVTZ basis set for $\mathrm{H}$ and $\mathrm{C}$ and the aug-cc-pVTZ for N, O and S.

Solvent effects (methanol) were included implicitly. The Conductor-like Polarizable Continuum Model $^{51}$ was used to calculate the electrostatic contribution to the free energy of solvation, whereas the non-electrostatic contributions were obtained using the SMD solvation model. ${ }^{52}$

Numerical integration of exchange-correlation functionals, both for geometry optimization and for the calculation of electronic transitions, was performed on a grid containing 99 radial points and 590 angular points around each atom. All the calculations were carried out using the GAMESS-US package. ${ }^{53,54}$ All figures were produced with the aid of the program wxMacMolPlt. ${ }^{55}$

\section{Acknowledgements}

This work was supported by CONICET, by ANPCyT and by UNLP of Argentina, R. P.-D., G. A. E., O. E. P. and B. S. P.-C. are members of the Research Career from CONICET.

\section{References}

1 E. Ritter, P. Przybylski, B. Brzezinski and F. Bartl, Curr. Org. Chem., 2009, 13, 241-249.

2 P. Przybylski, A. Huczynski, K. Pyta, B. Brzezinski and F. Bartl, Curr. Org. Chem., 2009, 13, 124-148.

3 P. A. Vigato, V. Peruzzo and S. Tamburini, Coord. Chem. Rev., 2012, 256, 953-1114.

4 Z. H. Chohan, S. H. Sumrra, M. H. Youssoufi and T. B. Hadda, Eur. J. Med. Chem., 2010, 45, 2739-2747.

5 I. Correia, J. Costa Pessoa, M. T. Duarte, M. F. Minas da Piedade, T. Jackush, T. Kiss, M. M. C. A. Castro, C. F. G. C. Geraldes and F. Avecilla, Eur. J. Inorg. Chem., 2005, 732-744.

6 M. J. Hearn, M. H. Cynamon, M. F. Chen, R. Coppins, J. Davis, H. J. O. Kang, A. Noble, B. Tu-Sekine, M. S. Terrot, D. Trombino, M. Thai, E. R. Webster and R. Wilson, Eur. J. Med. Chem., 2009, 44, 4169-4178.

7 K. C. Gupta and A. K. Sutar, Coord. Chem. Rev., 2008, 252, 1420-1450.

8 M. A. Hegazy, A. M. Hasan, M. M. Emara, M. F. Bakr and A. H. Youssef, Corros. Sci., 2012, 65, 67-76.

9 L. P. Singh and J. M. Bhatnagar, Talanta, 2004, 64, 313-319.

10 V. I. Minkin, A. V. Tsukanov, A. D. Dubonosov and V. A. Bren, J. Mol. Struct., 2011, 998, 179-191 and references therein.

11 M. Sliwa, S. Létard, I. Malfant, M. Nierlich, P. G. Lacroix, T. Asahi, H. Masuhara, P. Yu and K. Nakatani, Chem. Mater., 2005, 17, 4727-4735.

12 E. Hadjoudis and I. Mavridis, Chem. Soc. Rev., 2004, 33, 579-588.

13 S. D. Chatziefthimiou, Y. G. Lazarou, E. Hadjoudis, T. Dziembowska and I. M. Mavridis, J. Phys. Chem. B, 2006, 110, 23701-23709.

14 Tautomerism: Methods and Theories, ed. L. Antonov, Wiley-VCH, Weinheim, 2014.
15 K. Ogawa and J. Harada, J. Mol. Struct., 2003, 647, 211-216. 16 T. M. Krygowski, K. Woźniak, R. Anulewicz, D. Pawlak, W. Kolodziejski, E. Grech and A. Szady, J. Phys. Chem. A, 1997, 101, 9399-9404.

17 M. Rubčić, K. Užarević, I. Halasz, N. Bregović, M. Mališ, I. Dilović, Z. Kokan, R. S. Stein, R. E. Dinnebier and V. Tomišić, Chem. - Eur. J., 2012, 18, 5620-5631 and references therein.

18 M. Juribašić, N. Bregović, V. Stilinović, V. Tomišić, M. Cindrić, P. Šket, J. Plavec, M. Rubčić and K. Užarević, Chem. - Eur. J., 2014, 20, 17333-17345.

19 P. M. Dominiak, E. Grech, G. Barr, S. Teat, P. Mallinson and K. Woźniak, Chem. - Eur. J., 2003, 9, 963-970.

20 O. Dominguez, B. Rodriguez, M. Rodriguez, A. Ariza, N. Farfan and R. Santillan, New J. Chem., 2011, 35, 156-164.

21 H. Pizzala, M. Carles, W. E. E. Stone and A. Thevand, J. Chem. Soc., Perkin Trans. 2, 2000, 935-939.

22 A. C. González-Baró, R. Pis-Diez, B. S. Parajón-Costa and N. A. Rey, J. Mol. Struct., 2012, 1007, 95-101.

23 Q. Guo, L. Li, J. Dong, H. Liu, T. Xu and J. Li, Spectrochim. Acta, Part A, 2013, 106, 155-162.

24 X. Zhang, C. Bi, Y. Fan, Q. Cui, D. Chen, Y. Xiao and Q. Ping Dou, Int. J. Mod. Biol. Med., 2008, 22, 677-682.

25 J. Costa Pessoa, I. Cavaco, I. Correia, M. T. Duarte, R. D. Gillard, R. T. Henriques, F. J. Higes, C. Madeira and I. Tomaz, Inorg. Chim. Acta, 1999, 293, 1-11.

26 Z. Yin-Zhuang, L. Jin-Fang, T. Shu-Jie and Z. Min, Chin. J. Struct. Chem., 2005, 24, 1193-1197.

27 L. J. Farrugia, J. Appl. Crystallogr., 1997, 30, 565.

28 J. M. Fernández-G, F. del Rio-Portilla, B. Quiroz-García, R. A. Toscano and R. Salcedo, J. Mol. Struct., 2001, 561, 197-207 and references therein.

29 H. Karabiyik, B. Güzel, M. Aygün, G. Boğa and O. Büyükgüngör, Acta Crystallogr., Sect. C: Cryst. Struct. Commun., 2007, 63, 0215-0218.

30 F. H. Allen, A. Kennard, D. G. Watson, L. Brammer, A. G. Orpen and R. Taylor, J. Chem. Soc., Perkin Trans. 2, 1987, S1-S19.

31 K. Yvon, W. Jeitschko and E. Parthe, J. Appl. Crystallogr., 1977, 10, 73-74.

32 Y. Okaya, Acta Crystallogr., 1966, 21, 726.

33 K. Ohno, Y. Mandai and H. Matsuura, J. Mol. Struct., 1992, 268, 41-50.

34 A. C. González-Baró, R. Pis-Diez, C. A. Franca, M. H. Torre and B. S. Parajón-Costa, Polyhedron, 2010, 29, 959.

35 D. Lin-Vien, N. B. Colthup, W. G. Fately and J. G. Grasselli, Infrared and Raman Characteristic Frequencies of Organic Molecules, Academic Press, Boston, 1991.

36 R. A. Nyquist, Spectrochim. Acta, 1963, 191, 1655-1684.

37 J. W. Ledbettr, Jr, J. Phys. Chem., 1966, 70, 2245-2249.

38 N. Galić, D. Matković-Čalogović and Z. Cimerman, J. Mol. Struct., 1997, 406, 153-158.

39 N. Galić, Z. Cimerman and V. Tomišić, Spectrochim. Acta, Part A, 2008, 71, 1274-1280.

40 E. Pretsch, P. Bühlmann and M. Badertscher, Structure Determination of Organic Compounds, Springer, Berlin Heidelberg, 2009. 
41 R. M. Claramunt, C. López, M. D. Santa María, D. Sanz and J. Elguero, Prog. Nucl. Magn. Reson. Spectrosc., 2006, 49, 169-206.

42 A. Hidalgo, L. P. Avendaño Jiménez, L. A. Ramos, M. A. Mroginski, J. L. Jios, S. E. Ulic, G. A. Echeverría, O. E. Piro and E. Castellano, J. Phys. Chem. A, 2012, 116, 1110-1118.

43 CrysAlisPro, Oxford Diffraction Ltd., version 1.171.33.48 (release 15-09-2009 CrysAlis171.NET).

44 G. M. Sheldrick, Acta Crystallogr., Sect. A: Found. Crystallogr., 2008, 64, 112-122.

45 J. J. P. Stewart, J. Mol. Model., 2007, 13, 1173-1213.

46 MOPAC2012, J. J. P. Stewart, Stewart Computational Chemistry, Colorado Springs, CO, USA, http://OpenMOPAC.net, 2012.

47 Y. Zhao and D. G. Truhlar, Theor. Chem. Acc., 2008, 120, 215. 48 Y. Zhao and D. G. Truhlar, Acc. Chem. Res., 2008, 41, 157.
49 P. Elliott, F. Furche and K. Burke, Rev. Comput. Chem., 2009, 26, 91-165.

50 C. Adamo and V. Barone, J. Chem. Phys., 1999, 110, 6158-6170.

51 M. Cossi, N. Rega, G. Scalmani and V. Barone, J. Comput. Chem., 2003, 24, 669.

52 A. V. Marenich, C. J. Cramer and D. G. Truhlar, J. Phys. Chem. B, 2009, 113, 6378-6396.

53 GAMESS-US Version 11 August 2011 (R1).

54 M. S. Gordon and M. W. Schmidt, in Theory and Applications of Computational Chemistry, the First Forty Years, ed. C. E. Dykstra, G. Frenking, K. S. Kim and G. E. Scuseria, Elsevier, Amsterdam, 2005, pp. 1167-1189.

55 B. M. Bode and M. S. Gordon, J. Mol. Graphics Modell., 1998, 16, 133. 